\title{
Enhancing Patient Safety Through a Standardized Model of Physiologic Monitoring
}

$\overline{\text { Mary-Anne Davies and Heather Tales }}$

\begin{abstract}
The use of physiologic monitoring (e.g., cardiac monitoring) as an important component in providing safe patient care has escalated over the past two decades. It enables the clinician to detect physiologic changes in the patient's condition before they become clinically significant, thus allowing anticipation and prevention of adverse events. Issues and concerns regarding physiologic monitoring were raised throughout the London Health Sciences Centre (LHSC) leading to the approval of a project to develop a policy and guidelines for its use: the focus being standardization of processes and patient safety improvements. This article describes the underlying issues, the execution and results of the project, and its impact on patient safety within LHSC.
\end{abstract}

atient monitoring is pivotal for the appropriate assessment, diagnosis and treatment of patients. It ranges from basic vital signs and visual assessment to the use of sophisticated physiologic monitoring equipment that can measure a number of parameters such as cardiac rhythm, oxygen saturation and central venous and pulmonary artery pressures. Patients today present with higher acuity and are more complex in their care requirements, thus healthcare practitioners often rely on sophisticated monitoring technology. The use of physiologic monitoring enables the clinician to detect changes in a patient's condition before they become clinically significant, so that adverse outcomes to the patient can be anticipated and prevented. The progress of technology to include flexible monitoring has now made it preferable in some care settings to bring the technology to the patient instead of moving the patient to a critical care unit (Macready and Evans 1997). Modern monitoring systems are complex and require adequately trained staff to ensure the equipment is functioning properly and to be able to analyze monitoring data to prevent misdiagnoses (Drew et al. 2004). Regardless of where the patient is located, the process for monitoring the patient should be consistent. The assurance of patient safety depends on the appropriate, consistent and proper use of physiological monitoring.

Over the years, as our healthcare organization has expanded and changed, monitoring practices have not evolved to keep pace with the expansion in patient care areas or advances in technology. This article describes one healthcare organization's efforts to identify the underlying issues regarding monitoring, develop strategies to standardize monitoring practices and recog- 
nize the impact on patient safety within the organization.

\section{BACKGROUND}

London Health Sciences Centre (LHSC) is a large tertiary care teaching hospital with over 8,000 healthcare professionals located in southwestern Ontario. The organization has 744 acute care beds and is located on two sites throughout the city of London, Ontario. LHSC underwent a merger of two large hospital systems 10 years ago. In addition to this merger, the delivery of services changed to a program management format. As a result, personnel, practices, procedures and staff development were decentralized to the programs, unintentionally creating duplication of several different monitoring practices. Examination of LHSC's monitoring practices was triggered by the review of a coroner's report that involved monitoring and patient assessment at another large Canadian teaching hospital. As a result, a number of issues in the current practice of physiologic monitoring were raised through leadership and nursing practice committees. Concerns expressed were related to inadequate surveillance of monitoring equipment (e.g., continuous pulse oximetry, central ECG monitors) and the knowledge, skill and expertise of staff to appropriately respond to monitoring information and equipment, particularly in non-critical care areas.

These identified concerns prompted the establishment of an interdisciplinary task group with two part-time project leaders to lead the Monitoring Project. The mandate of this group was to develop evidence-based principles and guidelines for the use and practice of physiologic monitoring throughout the organization. The project methodology included distribution of an organizational survey, literature review including coroners' reports, review of standards, practices and education materials within and outside of LHSC, development of a physician consultant group and consultation with key stakeholders providing physiologic monitoring at LHSC.

\section{The Project \\ The Monitoring Inventory Survey}

In order to validate and fully understand the monitoring issues and explore current monitoring practices within the organization, a monitoring inventory survey was conducted. The aim of the survey was to determine the types of monitoring in place, the clinical areas using monitoring, the location of any pre-existing guidelines, the level of education in place, staffing patterns and responsibilities for monitoring. The survey was widely distributed by hospital mail and e-mail of an on-line survey link to all clinical areas throughout the organization. Due to the type of distribution, the response rate is unknown; however, the surveys returned represented all clinical areas within the organization. Several issues were identified or validated during the process, including lack of standard guidelines for monitoring, unclear responsibilities/ accountabilities for monitoring, surveillance
The implementation of the

monitoring standards hospital-

wide will ensure the care of all

patients will be consistent from

shift to shift

of central monitors, inadequate and inconsistent education of staff, lack of a standardized curriculum, unclear process for assessing impact of new monitoring equipment, inconsistent documentation practices, and inactivation of alarms and setting alarm parameters. Interestingly, almost $70 \%$ of respondents had concerns about monitoring, which supports the need for a standardized approach for safe monitoring practices.

\section{Framework}

It became apparent that there were numerous interpretations about physiologic monitoring and the type of care that was required for patients. Thus, a framework was developed to guide the creation of monitoring standards to support the healthcare practitioner when caring for patients requiring physiological monitoring. The goal of the framework was to ensure that there were appropriate conditions established for any type of monitoring in any patient care area, allowing every patient the provision of consistent, safe care. The framework included a definition of the type of physiologic monitoring, patient criteria, staffing and education requirements and healthcare team responsibilities. Each physiologic monitoring standard was created using this framework. In total, 16 standards were created within the organization, including bedside ECG, telemetry, pulse oximetry and arterial blood pressure. Specialized monitoring, such as fetal heart rate monitoring, was not included in the project as standards have already been established by national bodies. Clinical areas using specialized monitoring will be required to develop a monitoring standard using the monitoring framework to ensure that there is consistency for physiologic monitoring. The implementation of the monitoring standards hospital-wide will ensure the care of all patients will be consistent from shift to shift, regardless of changing healthcare practitioners.

\section{Practice Standards and Policy}

With a framework established to guide the work, a review of the literature, benchmarking within Ontario and the United States, and review of existing protocols enabled the task group members to develop standards for practice. Established guidelines were incorporated where appropriate and updated with evidence from the literature. Consultation with key stakeholders was an 
important component of the process and included respiratory therapists, staff nurses, clinical educators, advanced practice nurses and physicians. There were three physician consultants who worked closely with the project leaders to ensure that the standards would reflect appropriate practice. In addition, a policy has been developed that applies to all of the standards and provides the overriding principles to guide the practice of monitoring. The guiding principles that are inherent in the policy include the need to have patients reassessed after a specific period of time to ensure patients are being monitored appropriately, documentation and communication of monitoring data, clinical assessment of the patient, activation of appropriate alarm parameters and education for staff.

\section{Education}

In order to address the concerns regarding the inconsistencies with education, work is underway to develop standardized curricula for each of the monitoring standards. The learning materials are based on the education requirements stipulated in the practice standards and are derived from a combination of current learning packages and suggestions from the literature. Work on the standardized curricula for basic arrhythmia analysis and telemetry monitoring is in progress. Use of this learning material has been piloted in a variety of clinical programs and is undergoing revisions to ensure that it meets the needs of the learner and the requirements identified in the standards. All of the curricula will include a standardized test to assess competence. A separate group has developed learning material for pulse oximetry. Plans are to continue to develop standardized teaching materials for all types of monitoring and make those available in hard copy and as interactive on-line learning.

\section{Implications for Patient Safety}

The intent of the project was to develop monitoring practices to support patient safety. Examination of James Reason's Swiss Cheese Model of Defences (1997) provides some context for the issues identified and solutions developed for monitoring. Reason describes the use of barriers as a way of preventing potential hazards from resulting in a poor outcome. Latent conditions can produce holes in these barriers and thus weaken
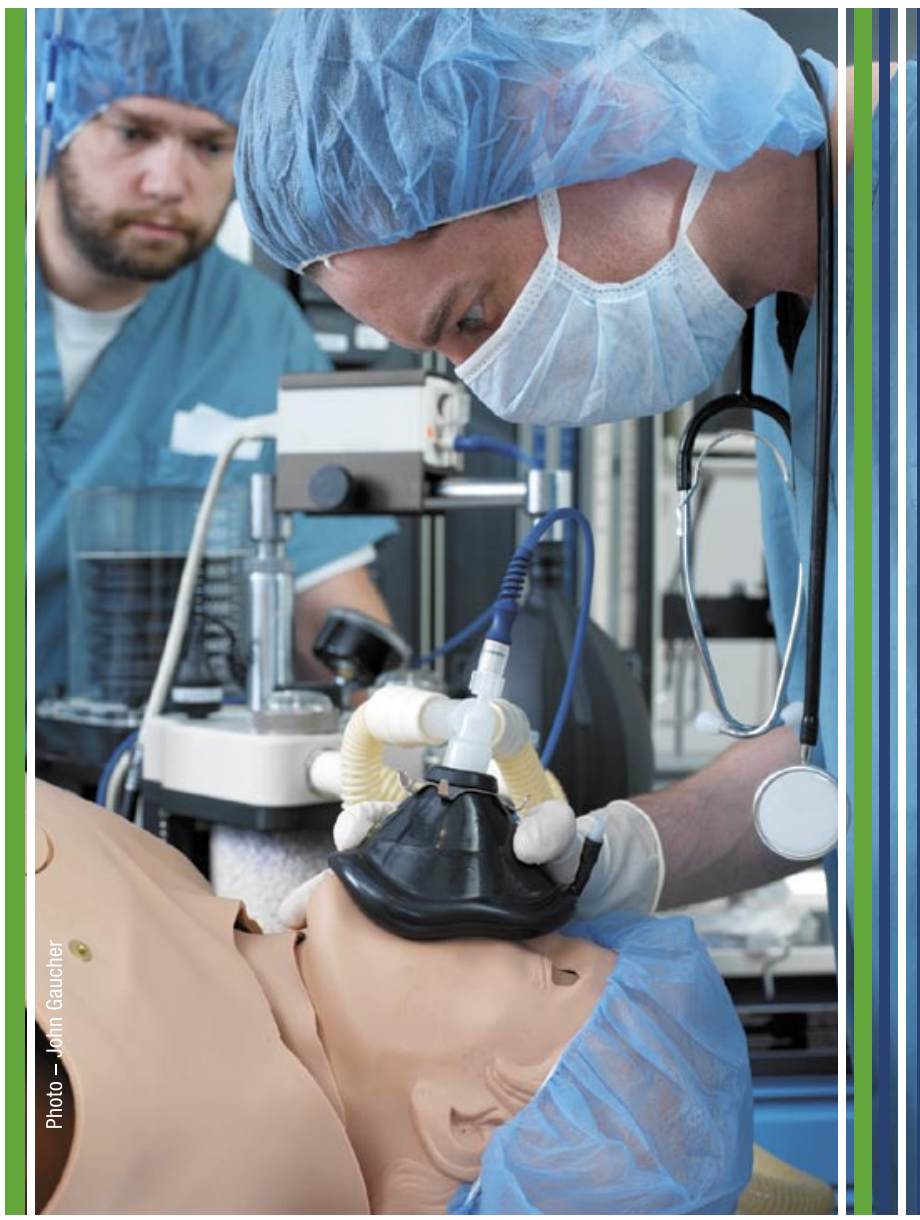

\section{$\checkmark$ Practice Improves Safety}

\section{Capital Health in Edmonton is committed to providing safe care for its patients, clients and residents. Simulation technology is just one of the many ways we're helping staff and physicians build a culture of safety.}

Capital Health's high-tech patient simulation program creates a realistic environment where multidisciplinary teams can practice procedures, gain experience in managing emergencies or unexpected events or engage in complex scenarios - before they encounter these situations in a real patient care setting.

\section{www.capitalhealth.ca}

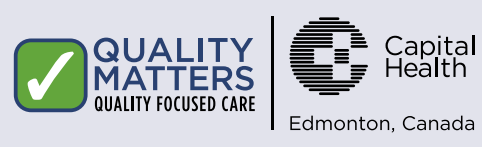


the defences. When an active failure, described as an unsafe act, is introduced into the system, the result can be devastating. Through the work of the Monitoring Project, the latent conditions related to physiologic monitoring at LHSC were identified. These included deficient or limited guidelines and policies, inconsistent training and practices and staffing issues. The lack of consistency in practice and education for monitoring resulted in confusion about appropriate monitoring practices. While there were no specific documented errors recorded at LHSC, there were likely near misses that had gone unreported. However, the potential for an adverse event was very real especially since latent conditions can create factors that promote errors (Reason 1997). Introduction of an unsafe act, such as deactivating an alarm and leaving the patient unattended, could result in an adverse event if a patient develops a lethal cardiac rhythm. Alternatively, lack of documentation or communication of significant findings

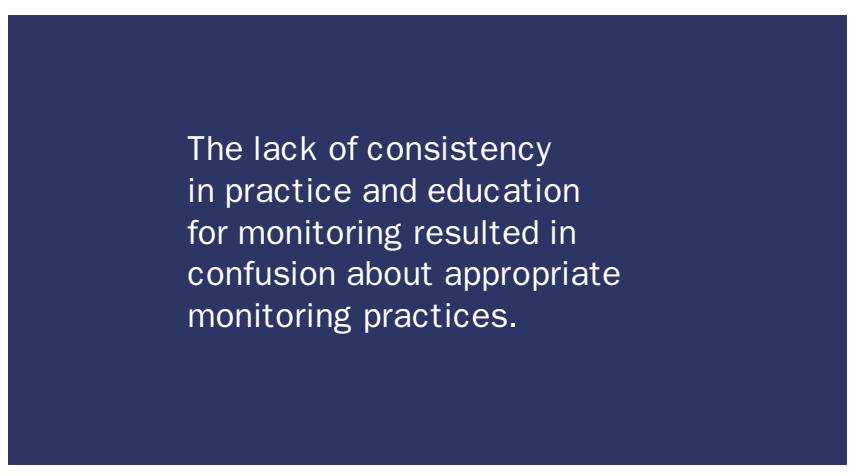

amongst care providers could lead to an inappropriate treatment plan and ultimately a negative outcome.

A standardized approach to monitoring practices and education will improve patient safety. Standardization is known to decrease the chance of errors because it limits the variety of methods in performing a task (Porto 2001). Adequate training that is planned, provided during non-work hours and allows for the appropriate interaction with a qualified educator is necessary to prevent errors. This is especially needed with advances in technology and high staff turnover (Porto 2001). Providing healthcare practitioners with the skills and knowledge for interpreting monitoring information and a process for monitoring patients will increase their capacity to respond to the information and decrease the opportunities for adverse outcomes (Walsh and Beatty 2002). Standardizing the approach to physiologic monitoring throughout the organization will also support the implementation of safety principles within LHSC (Kohn, Corrigan and Donaldson 1999).

\section{CONCLUSION}

The purpose of the Monitoring Project was to develop evidence- based principles and guidelines to ensure safe monitoring for all patients. This was achieved by developing a standardized approach to physiologic monitoring that includes practice standards, a corporate policy and standardized education. It is clearly articulated in the policy and standards that monitoring is an adjunct to patient care and to be used as a tool in assisting clinicians with their assessment of the patient. While it is recognized that removing hazards is a more effective way of preventing errors, the development of policies, procedures and staff education are necessary to address the latent conditions that can weaken our defensive barriers (Reason 1997). By addressing these issues in the policy and standards, LHSC will strengthen the defences necessary to ensure the hazards of physiologic monitoring do not result in an adverse event.

\section{Acknowledgements}

The authors of the paper would like to acknowledge Margaret Nish, Executive Vice-President, Clinical Care and Academic Affairs, Dr. Ian Herrick, Chief of Staff, Dr. Murray Girotti, Senior Medical Advisor, Carol Wong, RN, MScN and the members of the Monitoring Guidelines Task Force for their contributions, guidance and support provided to this initiative. They were instrumental in identifying the monitoring issues within the organization and lobbying for a plan to allocate the appropriate resources to support monitoring practices.

\section{References}

Drew, B.J., R.M. Califf, M. Funk, E.S. Kaufman, M.W. Krucoff, M.M. Laks, P.W. Macfarlance, C. Sommargren, S. Swiryn, G.F. Van Hare. 2004. "AHA Scientific Statement. Practice Standards for Electrocardiographic Monitoring in Hospital Settings." Circulation 110: $2721-46$.

Kohn, L.T., J.M. Corrigan, M.S. Donaldson. 1999. "Institute of Medicine (IOM) Committee on Quality of Health Care in America." To Err is Human: Building a Safer Health System. Washington, DC: National Academy Press.

Macready, N. and A. Evans. 1997. "Flexible Monitoring: Mobilizing Critical Care.” American Journal of Critical Care 6(4): 3-15.

Porto, G.G. 2001. "Safety by Design: Ten Lessons From Human Factors Research.” Journal of Healthcare Risk Management Fall: 43-50.

Reason, J. 1997. Managing the Risks of Organizational Accidents. Hampshire, England: Ashgate Publishing Limited.

Walsh, T. and P.C.W. Beatty. 2002. "Human Factors Error and Patient Monitoring." Physiologic Measurement 23: R111-R132.

\section{About the Authors \\ Mary-Anne Davies, MScN, RN, is Patient Safety Specialist, Quality and Patient Safety at London Health Sciences Centre, London, Ontario, Canada.}

Heather Tales, BScN, RN, is Nursing Professional Practice Specialist at London Health Sciences Centre, London, Ontario, Canada.

Corresponding Author: Mary-Anne Davies, Email: maryanne. 